Revista Colombiana de Obstetricia y Ginecología Vol. 57 No. 3 • 2006 • (207-2 10)

Serie de CAsos

\title{
EMBRIOSCOPÍA EN ABORTO RETENIDO
}

\section{Embrioscopy in missed abortion}

\author{
Jesús Alberto Ruíz-Ruíz, M.D.*, Eugenia Corredor-Sánchez, M.D. **, Carlos Julio \\ García-Perlaza, M.D.***, José Ignacio Madero-Cervera, M.D.****, Claudia Rocío \\ López, B.Sc ${ }^{+}$, María Fernanda León, B.Sc ${ }^{++}$Magally Escobar, B.Sc ${ }^{+++}$ \\ Recibido: enero 31/06 - Revisado: marzo 21/06 - Aceptado: agosto 16/06
}

\section{RESUMEN}

Objetivo: describir los hallazgos por embrioscopía transcervical en pacientes con aborto retenido, de diferentes semanas de gestación en primer trimestre.

Institución: Medifértil, Bogotá, Colombia.

Materiales y métodos: estudio descriptivo tipo serie de casos. Pacientes con aborto retenido diagnosticado por ecografía; se describe el cariotipo de la muestra obtenida por legrado obstétrico posterior al procedimiento de embrioscopía.

Resultados: el procedimiento fue realizado exitosamente a las seis pacientes, cuatro de las cuales presentaron alteraciones fenotípicas todas con cariotipo normal, un caso presentó trisomía 21 sin alteraciones fenotípicas y un caso en que se identificó degeneración hidrópica, correspondió a una mola parcial.

Conclusión: la embrioscopía transcervical previa al legrado obstétrico podría ser una alternativa diagnóstica útil, principalmente en pacientes con pérdida temprana posterior a reproducción asistida y pérdida recurrente de la gestación.

\footnotetext{
Medifértil: Programa de Medicina Reproductiva.

Calle 127 \# 28 - 75. Bogotá. Colombia. medifertil@yahoo.com

Director General. Medifertil Programa de Medicina Reproductiva.

Bogotá, Colombia. Correo electrónico: medifertil@yahoo.com

** Residente de 3er año de Ginecología y Obstetricia. Hospital la Samaritana. Bogotá, Colombia.

*** Residente de 3er año de Ginecología y Obstetricia. Hospital la Samaritana. Bogotá, Colombia.

**** Director Científico. Medifertil Programa de Medicina Reproductiva Bogotá, Colombia.

Bacterióloga, Embrióloga. Laboratorio de Reproducción Asistida. Medifertil. Bogotá, Colombia.

++ Bacterióloga, Embrióloga. Laboratorio de Reproducción Asistida. Medifertil. Bogotá, Colombia.

+++ Bacterióloga. Inmunóloga. Centro de Referencia en Infertilidad y Autoinmunidad. CERIA.
}

Palabras clave: embrioscopía, aborto retenido, embrión, feto, cariotipo, vellosidad coriónica.

\section{SUMMARY}

Objective: showing the early experience and the findings in six patients suffering missed miscarriage having different weeks of gestation, who received transcervical embrioscopy before obstetric curettage whilst receiving the same anesthesia.

Institution: Medifértil, Bogotá, Colombia. Type of Study: descriptive series of cases.

Materials and methods: kariotyping and transcervical embrioscopy was carried out on each of the six patients having a previous diagnosis of missed miscarriage, confirmed by transvaginal ultrasonography during the first three months.

Results: transcervical embrioscopy was successfully done on all the patients; four of them (66\%) exhibited phenotype abnormalities, every case displayed a normal kariotype (analysis of 30 methaphases). One of the cases expressed a trisomy 21 and the other corresponded to hydropic degeneration related to partial mole.

Conclusion: We suggest applying transcervical embrioscopy before obstetric curettage in patients diagnosed as having early and recurrent loss as a complementary tool for identifying genetic abnormalities. It is also a procedure having low morbidity and uses the same hysteroscopy infrastructure.

Key words: embrioscopy, missed abortion, embryo, foetus, karyotype, chromosomal abnormality, chorionic villus. 


\section{INTRODUCCIÓN}

La tasa de pérdidas de embarazos tempranos se acerca al 50\%, sin embargo solo un bajo porcentaje de estos son reconocidos clínicamente, El 80\% de los abortos espontáneos ocurren en las primeras 12 semanas. Casi el 70\% de estos se deben a anormalidades cromosómicas tales como: trisomías, monosomías de cromosoma sexual y poliploidías, asociadas a alteraciones fenotípicas del embrión. ${ }^{1}$ Existen otras alteraciones en el embrión causadas por causa extrínseca como bandas, miomas u oligohidramnios.

Dado que el estudio de las alteraciones cromosómicas se hace mediante el análisis citogenético convencional del cultivo de tejido ovular que depende de la calidad y cantidad de la muestra obtenida, la mala calidad del tejido obtenido incrementará la tasa de fallas del mismo.

La embrioscopía es una técnica invasiva para la visualización directa del embrión o del feto temprano (figura 1); proporciona una oportunidad única para examen del desarrollo embrionario y ofrece el potencial para diagnóstico y/o intervención terapéutica. Permite identificar anomalías estructurales y obtener directamente muestra de sangre fetal, tejido y líquido amniótico. ${ }^{2}$ (Figuras 2,3).

La embrioscopía transcervical en casos de aborto retenido permite visualización directa del embrión muerto in utero, no afectado por el daño causado por la evacuación instrumental o el pasaje espontáneo; por esto ha sido sugerida como estudio complementario en la etiología del aborto retenido, una vez este ha sido

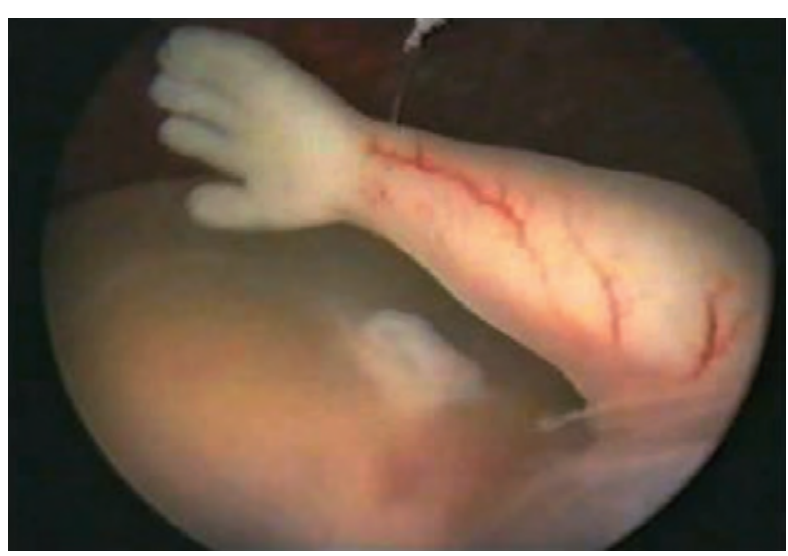

Figura 1. Miembro superior izquierdo. Polo cefálico. confirmado por hallazgo ecográfico de embrión con ausencia de actividad cardiaca, cuando la longitud del embrión excede la longitud discriminatoria, que corresponde a una longitud embrionaria de $5 \mathrm{~mm}$. ${ }^{3,4}$

El objetivo de este estudio es informar los hallazgos encontrados en seis pacientes con diagnóstico de aborto retenido a quienes se les practicó embrioscopía transcervical.

\section{MATERIALES Y MÉTODOS}

Diseño: serie de casos prospectiva.

Población: pacientes con aborto retenido confirmado por ecografía atendidas en Medifértil, institución privada de referencia en estudios de infertilidad, con un programa de medicina reproductiva ubicada en Bogotá (Colombia), entre febrero 2004 y julio de 2005 las cuales fueron sometidas a embrioscopía transcervical, antes de realización de legrado obstétrico.

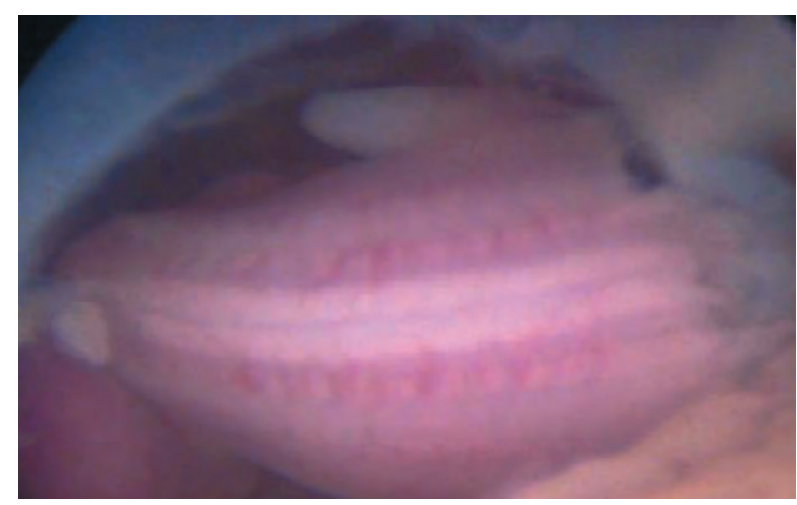

Figura 2. Anencefalia en embrión de 7 semanas.

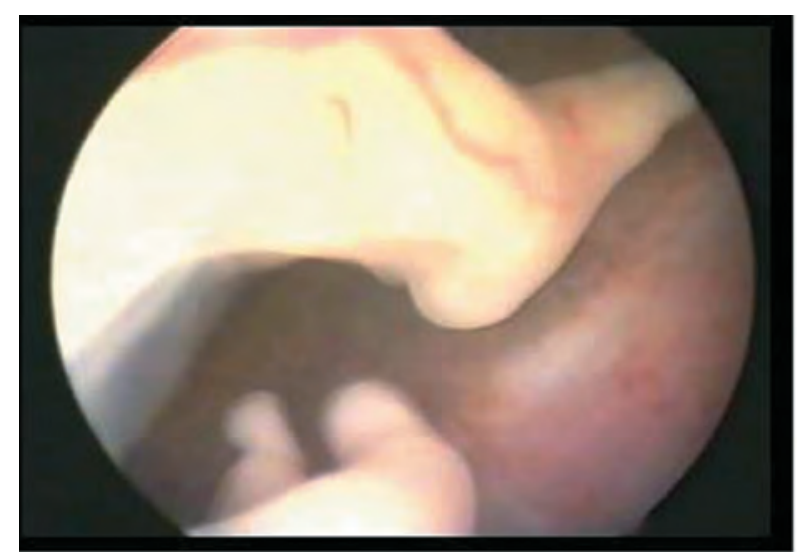

Figura 3. Sindactilia en pie derecho en embrión de 10 semanas. 
El procedimiento se realizó bajo anestesia general, con histeroscopio rígido marca Storz ${ }^{\circledR}$ de 5,9 mm y 30 grados de ángulo visual, con canal operatorio de $2 \mathrm{~mm}$ conectado a monitor y unidad de video. Se usó medio de distensión hídrico con solución salina a 40 - 120 mm Hg. de presión, debido a que ofrece una adecuada distensión, con óptima visualización.

La técnica fue desarrollada con la paciente en posición de litotomía; se realizó especuloscopía, pinzamiento de labio anterior del cérvix y paso de histeroscopio a través de él, se ingresó a la cavidad endometrial donde se identificaron la decidua parietal y la superficie de la cavidad; posteriormente se observó el sitio de implantación, se evaluó la decidua capsular y se disecaron las membranas ovulares en forma cortante con microtijera a través del canal operatorio; logrando ingresar a la cavidad amniótica, la cual se evaluó en conjunto con el corion y el embrión

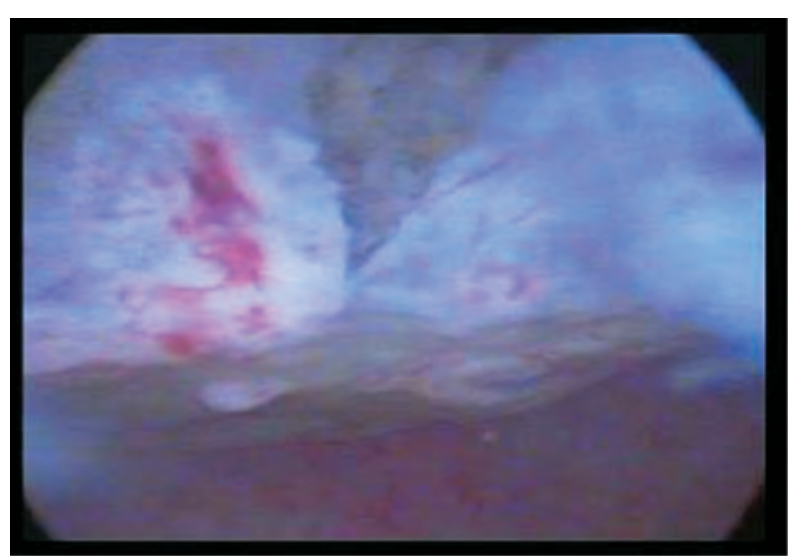

Figura 4. Apertura de corion, aborto retenido de 7 semanas.

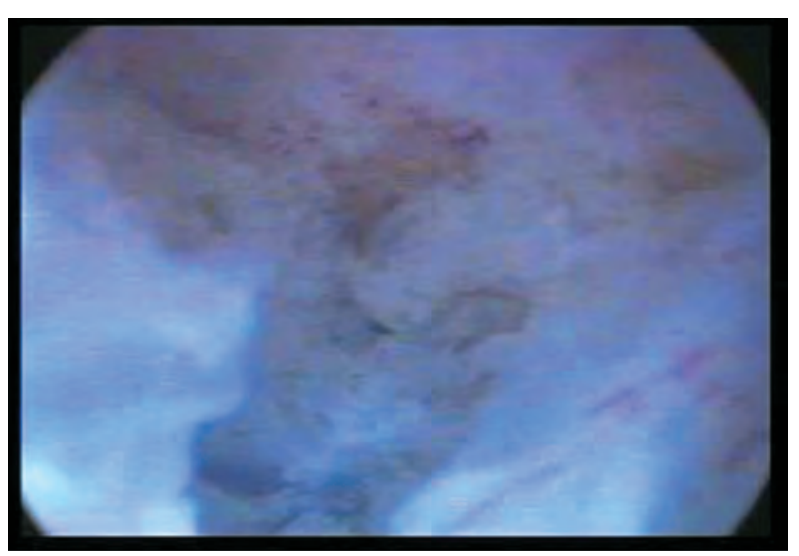

Figura 5. Corion frondoso, 7 semanas.

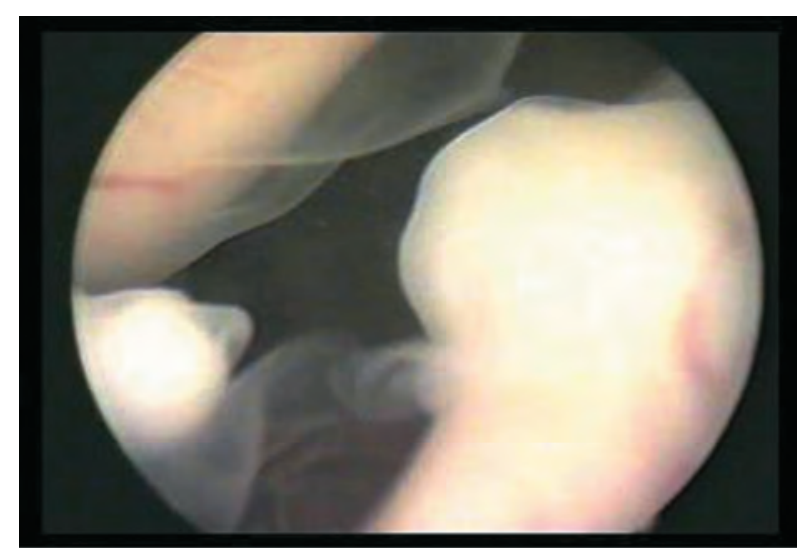

Figura 6. Genitales externos, sexo masculino.

(figuras 4 y 5). Se detalló la anatomía embrionaria (figura 6) y se comparó para diferenciar los hallazgos anormales y la edad gestacional con la tabla de estadio de desarrollo embrionario por embrioscopía de Carnegie. Se practicó estudio con cariotipo al producto de legrado realizado inmediatamente después de la embrioscopía.

\section{RESULTADOS}

El procedimiento fue realizado exitosamente en 6 pacientes incluidas en el estudio. En todos los casos fue posible visualizar la cavidad uterina, disecar las membranas e inspeccionar la cavidad amniótica y el trofoblasto. En un caso no se identificó embrión.

En cuatro pacientes se observaron anormalidades fenotípicas del embrión consistentes en: dos gastrosquisis, una amelia, una aracnodactilia y el quinto embrión fue fenotípicamente normal. En el caso donde no se identificó embrión se observó degeneración hidrópica del trofoblasto.

El estudio de cariotipo mostró que cuatro embriones con alteraciones morfológicas presentaban cariotipo normal y el embrión con fenotipo normal mostró trisomía 21 en el estudio genético, el caso de la embrioscopía con trofoblasto hidrópico presentó triploidía.

No se presentaron complicaciones propias del procedimiento, ni en el postoperatorio. El tiempo promedio de duración de la embrioscopía fue 10 minutos. Ninguna de las anormalidades detectadas durante la embrioscopía fue visualizada previamente por ultrasonido. 


\section{DISCUSIÓN}

Este estudio muestra la primera serie de casos de embrioscopía reportada en nuestro país, nos sugiere que la embrioscopía podría ser un elemento a tener en cuenta en el diagnóstico de la paciente con aborto retenido.

T. Philipp y colaboradores realizaron un estudio de 272 abortos, sometidos a embrioscopía transcervical, de los cuales se logró visualización exitosa del embrión o del feto temprano en 233 casos, se realizó cariotipo a 221. De los casos sometidos a análisis citogenético, 165 (75\%) presentaron cariotipo anormal, de estos, 150 (91\%) presentaban desarrollo morfológico anormal. 56 (25\%) casos cursaron con cariotipo normal, pero solamente $16(28,5 \%)$ presentaban desarrollo morfológico normal. ${ }^{3}$ La baja frecuencia de alteraciones del cariotipo encontrada en nuestra investigación, podría ser explicada por el pequeño número de pacientes, o pudo ser debido a que las muestras para el análisis citogenético no fueron tomadas directamente del embrión y del trofoblasto, sino del legrado obstétrico posterior a la embrioscopía. El manejo convencional de estas pacientes con legrado, dificultaría la obtención de material adecuado para la detección de anormalidades cromosómicas estructurales debido a fragmentación del embrión y contaminación por células maternas que no permiten una evaluación del fenotipo y la identificación de malformaciones localizadas. ${ }^{5}$

En otro estudio realizado por Philipp T. y colaboradores ${ }^{6}$ se observó un hallazgo significativo de alteraciones trofoblásticas no identificadas por ecografía en aborto retenido, principalmente molas parciales; en nuestro estudio observamos 1 caso de degeneración hidrópica.

La embrioscopía en casos de aborto retenido puede revelar anormalidades morfológicas no detectables por ultrasonido, además en especímenes de aborto con cromosomas aparentemente normales podría servir como punto de partida para la realización de estudios genéticos más detallados, que permitan una mejor comprensión de la etio-logía de las pérdidas espontáneas en embarazos tempranos. ${ }^{3,6,7}$
Es motivo de controversia la recomendación de realización de embrioscopía y estudios citogenéticos a todas las mujeres con aborto retenido por tratarse de una patología con bajo riesgo de recurrencia, por ser un procedimiento invasivo y que además implica un costo extra en el manejo de estas pacientes; sin embargo es indiscutible la ventaja de proveer datos de comprensión etiológica en aborto temprano. ${ }^{3}$

En parejas con historia de pérdida recurrente del embarazo un examen embrioscópico detallado es probable que sea útil, ya que en estos casos el análisis cromosómico es generalmente recomendado y puede proveer información de gran valor para consejería genética. ${ }^{3}$

\section{REFERENCIAS}

1. Speroff L, Fritz MA. Recurrent Early Pregnancy Loss. En: Clinical Gynecologic Endocrinology and Infertility. 7th ed. Philadelphia: Lippincott Williams \& Wilkins; 2004. p. 1069-101.

2. Cullen MT, Reece A, Whetham JD, Hobbins JC. Embryoscopy: description and utility of a new technique. Am J Obstet Gynecol 1990;162:82-6.

3. Philipp T, Philipp K, Reiner A, Beer F, Kalousek DK. Embryoscopic and cytogenetic analysis of 233 missed abortions: factors involved in the pathogenesis of developmental defects of early failed pregnancies. Hum Reprod 2003;18:1724-32.

4. Laing FC, Frapes MC. Evaluación ecográfica durante el primer trimestre del embarazo. En: Callen PW. Ecografía en Obstetricia y Ginecología. 4a. edición. Buenos Aires: Editorial Médica Panamericana; 2002. p. 126-34.

5. Lomax B, Tang S, Separovic E, Phillips D, Hillard E, Thomson T, et al. Comparative genomic hybridization in combination with flow cytometry improves results of cytogenetic analysis of spontaneous abortions. Am J Hum Genet 2000;66:1516-21.

6. Philipp T, Kalousek DK. Generalized abnormal embryonic development in missed abortion: embryoscopic and cytogenetic findings. Am J Med Genet 2002;111:43-7.

7. Ferro J, Martinez MC, Lara C, Pellicer A, Remohi J, Serra V. Improved accuracy of hysteroembryoscopic biopsies for karyotyping early missed abortions. Fertil Steril 2003;80:1260-4. 